To AppeAR IN ApJ LetTers

Preprint typeset using $\mathrm{LT}_{\mathrm{E}} \mathrm{X}$ style emulateapj v. 5/2/11

\title{
2FGL J1311.7-3429 JOINS THE BLACK WIDOW CLUB
}

\author{
ROGER W. ROMANi \\ Department of Physics, Stanford University, Stanford, CA 94305 \\ To Appear in ApJ Letters
}

\begin{abstract}
We have found an optical/X-ray counterpart candidate for the bright, but presently unidentified, Fermi source 2FGL J1311.7-3429. This counterpart undergoes large amplitude quasi-sinusoidal optical modulation with a $1.56 \mathrm{~h}(5626 \mathrm{~s})$ period. The modulated flux is blue at peak, with $T_{\text {eff }} \approx 14,000 \mathrm{~K}$, and redder at minimum. Superimposed on this variation are dramatic optical flares. Archival X-ray data suggest modest binary modulation, but no eclipse. With the $\gamma$-ray properties, this appears to be another black-widow-type millisecond pulsar. If confirmation pulses can be found in the $\mathrm{GeV}$ data, this binary will have the shortest orbital period of any known spin-powered pulsar. The flares may be magnetic events on the rapidly rotating companion or shocks in the companion-stripping wind. While this may be a radio-quiet millisecond pulsar, we show that such objects are a small subset of the $\gamma$-ray pulsar population.
\end{abstract}

Subject headings: gamma rays: stars — pulsars: general

\section{INTRODUCTION}

In the most recent Fermi Large Area Telescope (LAT) catalog of $18730.1-100 \mathrm{GeV}$ sources, over 1170 have statistically reliable counterpart identifications at lower energy (Nolan et al. 2012). The bulk of these identifications are blazars and spin-powered pulsars, with young pulsars (both radio-selected and Geminga-like $\gamma$-ray selected) dominating at low Galactic latitude. An additional population of millisecond pulsars extends to high latitude among the blazarassociated sources. Identification progress is especially impressive for the bright, well localized sources that have been studied since early in the Fermi mission. Here we update Abdo et al. (2009a), selecting 'bright' sources from the 2FGL catalog as those with $>20 \sigma$ detection significance and timeaveraged energy flux $F_{E}>3 \times 10^{-11} \mathrm{erg} \mathrm{cm}^{2} \mathrm{~s}^{-1}-$ there are 249 such sources. All but six presently have lower energy identifications: blazars, pulsars and a few binaries. The handful of sources not yet associated with one of these source classes provide the best prospect for new types of $\gamma$-ray emitter. We have initiated a campaign to characterize these unidentified sources. The first result from this effort is the discovery of dramatic optical and X-ray variability for the high latitude $\left(|b|=62^{\circ}\right)$ source J2339-0533, implying that it is a short period 'black-widow'-type binary millisecond pulsar (MSP) (Romani \& Shaw 2011; Kong, et al. 2012).

Here we report progress on the next high latitude unidentified source in this set, 2FGL J1311.7-3429 (hereafter J1311) at $|b|=28^{\circ}$. With a $43 \sigma$ detection significance (the highest among the 2FGL unidentified sources) and an energy flux of $F_{0.1-100 G e V}=6.2 \times 10^{-11} \mathrm{erg} \mathrm{cm}^{-2} \mathrm{~s}^{-1}$ (the second brightest UnIDed 2FGL), this is a top candidate for followup. The 'Variability index' value 19.5 and 'Curvature Significance' $=6.3$ mark this as a steady source with a substantial spectral cut-off: a prime pulsar candidate. It has been searched for $<30 \mathrm{~Hz} \gamma$-ray pulsations (eg. Abdo et al. 2009b)

\footnotetext{
${ }^{1}$ Visiting Astronomer, Kitt Peak National Observatory and Cerro Tololo InterAmerican Observatory, National Optical Astronomy Observatory, which is operated by the Association of Universities for Research in Astronomy (AURA) under cooperative agreement with the National Science Foundation. The WIYN Observatory is a joint facility of the University of Wisconsin-Madison, Indiana University, Yale University, and the National Optical Astronomy Observatory.
}

and for radio pulses down to $\sim$ ms periods (Ray et al. 2012), with no detection. Thus it is unlikely to be an isolated young pulsar or a radio-loud MSP. We describe here an optical campaign to identify and characterize a counterpart.

\section{OBSERVATIONS}

Initial exposures of the J1311 error region were taken with the MiniMo camera at the $3.6 \mathrm{~m}$ WIYN telescope on Feb. 1718, 2012. Exposures were $180 \mathrm{~s}$ using the Gunn filter set $(2 \times$ $g, r, i$ on $2 / 17 ; 5 \times g, r$ on $2 / 18)$. Observations were perforce at airmass $2.5-3$, seeing was variable and conditions were non-photometric.

A more extensive image sequence was taken with the SOAR Optical Imager (SOI) at the $4.2 \mathrm{~m}$ SOAR telescope on March 21-23, 2012. Here $120 \mathrm{~s}$ exposures using SDSS filters were taken, covering one color each night $\left(61 \times g^{\prime}\right.$ on $3 / 21$, $74 \times r^{\prime}$ on $3 / 22$ and $57 \times i^{\prime}$ on $3 / 23$ ). The camera was binned $2 \times 2$, allowing fast $11 \mathrm{~s}$ read-out and high observation efficiency. Again image quality was highly variable (partly due to loss of wavefront sensor control of the primary).

We searched image archives for exposures covering J1311, finding a sequence (program 086.D-0388) of $170 \mathrm{~s}$ VLT/VIMOS exposures taken on March $4(2 \times[3 B, 3 \mathrm{~V}, 3 \mathrm{I}])$ and March 11, $2011(3 \times[3 B, 3 V, 3 I])$. VIMOS is an array camera with $2^{\prime}$ gaps between the four CCD chips, so the five pointings were offset to cover the full J1311 error region.

In examining the images for variable objects, we paid particular attention to sources coincident with detections in archival X-ray data (see below). One such star was blue and highly variable on short timescales. The NOMAD position of this likely counterpart is $131145.741,-343029.96$ (2000.0). While we ensured that this star was covered in all MiniMo and SOI exposures, we found that the counterpart was in the array gap for the first VIMOS pointing on March 3, 2011 and at the extreme edge of a chip, suffering major vignetting, in the second pointing on March 11, 2011.

To quantify the variations, we performed simple aperture photometry of the counterpart and surrounding field stars in all frames. Since the bulk of the imaging was in the SDSS filter set, we attempted to reference all photometry to this scale. The MiniMo data were calibrated using $g r i$ images in SDSS fields at low airmass; we transferred this calibra- 


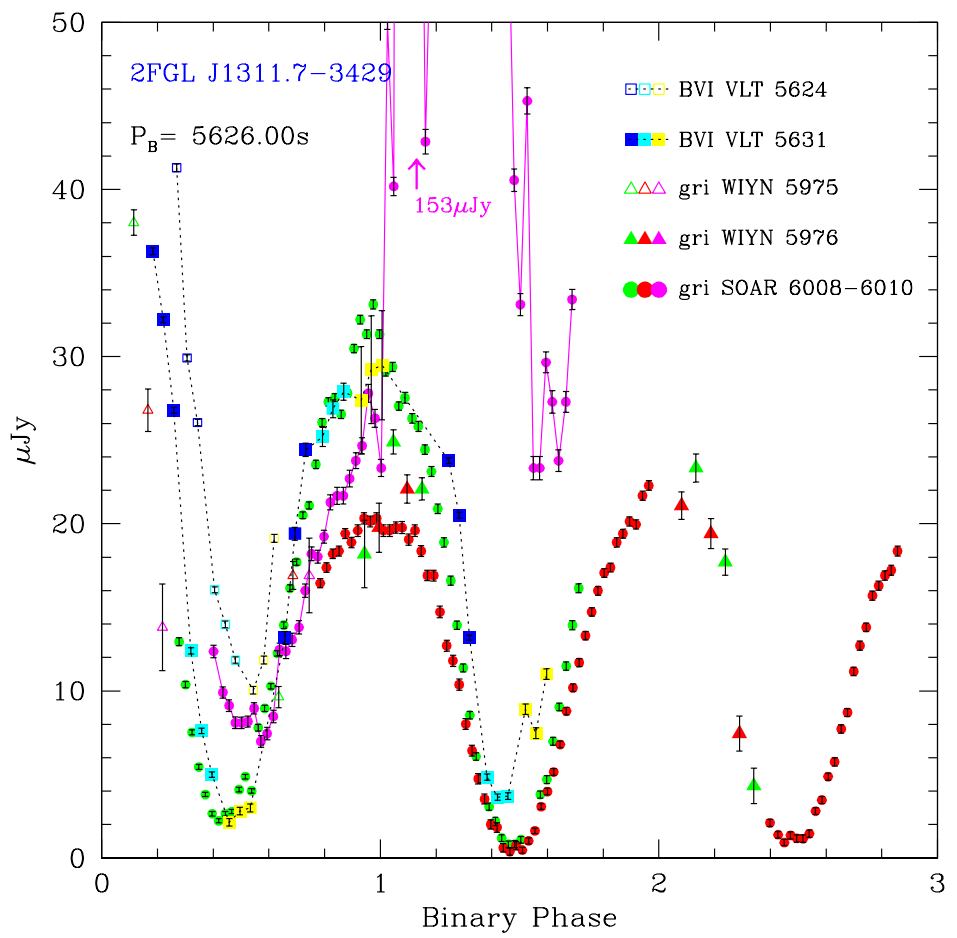

FIG. 1.- SOAR/WIYN/VLT photometry of J1311.7-3429. The abscissa is the phase relative to phase 0 (superior conjunction, secondary flux maximum) just before the first observation of each plotted night, using the best-fit $P_{b}=5626.00 \mathrm{~s}$ period. Symbols and colors indicate the various telescopes and observing bands. Photometry from the SOAR $i^{\prime}$ flare is connected with a solid line; points for each VLT night are connected with a dash line. tion to stars in the J1311 field. Unfortunately, the nonphotometric WIYN conditions and pass-band differences between the SDSS and Gunn sets make the substantial atmospheric extinction corrections for J1311 uncertain. This is not a severe problem for the field stars, which were mostly late type with $g^{\prime}-r^{\prime}=0.7-1.5$. However the target's color is as blue as $g^{\prime}-r^{\prime}=-0.5$ and varies appreciably with magnitude. Thus while the relative photometry for each color sequence has very small statistical errors, there may be a systematic offset between the WIYN and SOAR points as large as $0.2 \mathrm{mag}$, especially in $g^{\prime}$. For the VIMOS images, we used NOMAD field stars to establish zero points in B and $\mathrm{V}$. NOMAD-detected stars were generally saturated in the VIMOS I images, so we established the I scale using stars from the DENIS catalog.

We show the photometry for each night in Figure 1, converting to fluxes and plotting against estimated binary phase. The SOI $g^{\prime}, r^{\prime}$ data show dramatic quasi-sinusoidal variability by a factor $\sim 30 \times$ with a period of $\sim 1.5 \mathrm{~h}$. In $i^{\prime}$ an initial flux minimum was followed by a large $\sim 5 \times$ flare, with rapid fluctuations during return to quiescence. The $g^{\prime}, r^{\prime}$ fluxes also show variability, especially near maximum. The WIYN data are sparse and have larger statistical errors, but do follow the periodic modulation. Most WIYN fluxes appear $10-20 \%$ lower than SOAR at the corresponding phase, suggesting an underestimate of $\sim 0.1 \mathrm{mag}$ for the extinction correction. However, given the apparent non-periodic variability this cannot be firmly concluded. The VLT fluxes from March 11 pass through two minima; those from March 4 show one minimum, but appear to have $\sim 8 \mu \mathrm{Jy}$ additional flux.

\subsection{Orbital Period Estimate}

The intermittent flaring activity of J1311 compromises simple periodogram or Fourier methods for refining the period.
However, the combination of SOAR, WIYN and VLT exposures give photometry information on $1-3,7, \sim 35$, and $\sim 350$ day time scales, which suffices to resolve possible aliases. After referencing all observation mid-point times to the solar system barycenter, we can constrain the phase of the 2012 epoch minima to $\delta \phi \approx 0.02$, giving a best fit period of $P_{b}=5626.00 \pm 0.02 \mathrm{~s}$. The epoch of (quiescent) maximum light associated with our SOAR $r^{\prime}$ measurements (presumably pulsar superior conjunction, see below) is barycentric MJD 56009.1795 \pm 0.0013 .

The approximately sinusoidal nature of the light curve and very large modulation amplitude can be best interpreted in terms of strong companion heating. The inferred heating luminosity is appreciably above any X-ray flux (see below); we infer that accretion power is not dominant and that the heating source is invisible in the optical and faint in the X-ray. We conclude that this is a 'black-widow' type pulsar binary and is the likely counterpart of the $\gamma$-ray source. In this it is remarkably similar to the black widow candidate counterpart for $\mathbf{J} 2339-0533$. But the $\sim 3 \times$ shorter orbital period is unprecedented; this is an extreme black widow system.

\subsection{Archival $X$-ray Light Curve}

2FGL J1311.7-3429 has been observed by both the $C X O$ and Suzaku satellites. One exposure from each facility is available in the NASA archives, additional exposure has been taken but is not publicly available. The counterpart was detected in both data sets and well localized by $C X O$. We analyzed both observations for X-ray spectrum and variability.

The Suzaku observation (ObsID 804018010; Kataoka, PI) started on August 042009 (MJD 55047.2290) and had 33.4 ks of on-source time over $95 \mathrm{ks}$ ( $\sim 17$ binary orbits) with exposure in XIS0,1,3. A comparably bright source lies $\sim 1.6^{\prime}$ from the target, so we extracted source counts from a $1^{\prime}$ radius aperture. Figure 2 (left) shows the combined XIS count rate, both direct aperture counts (histogram) and the backgroundsubtracted aperture-corrected count rate (points). The vertical dotted lines mark the phases of maximum optical light; we see that orbital modulation is difficult to measure since $P_{b}$ is so close to that of satellites in low Earth orbit. A clear X-ray flare is seen $5000 \mathrm{~s}$ after the observation start.

An ACIS-I exposure of $19.8 \mathrm{ks}$ live-time (ObsID 11790; Cheung, PI) was taken starting MJD 5527.6652. Unfortunately, as for VLT observation 1, the counterpart was directly in the gap between ACIS-I chips. However, the $C X O$ dither did provide some ( $\sim 25 \%$ effective live-time) exposure and a source coincident with the optical counterpart was well detected with 60 counts. There is no strong evidence for secular X-ray variability on $3000 \mathrm{~s}$ timescales. The exposure only covers $\sim 3.5$ orbits. Figure 2 (right) shows the folded X-ray light curves from the Suzaku and $C X O$ data sets. After the early flare in the Suzaku data is excluded there is only weak evidence for orbital modulation; note there is an exposure gap for phases $\phi_{B}=0.7-0.8$. Similarly the $C X O$ data show no strong peak or eclipse. In both light curves there seems to be a slight excess at phases 0.7-1.1. This may indicate additional weaker flaring activity near superior conjunction.

Only crude spectral constraints can be obtained with the limited counts, especially after selecting for Suzaku photons outside of the flare period. If we fit an absorbed power law to the X-ray data we find that $N_{H}$ is essentially unconstrained $\left(0.5 \pm 6 \times 10^{21} \mathrm{~cm}^{-2}\right)$ but consistent with the limited extinction in this high $|b|$ direction. We therefore fix at the $N_{H}=5 \times 10^{20} \mathrm{~cm}^{-2}$ inferred from the dust maps 

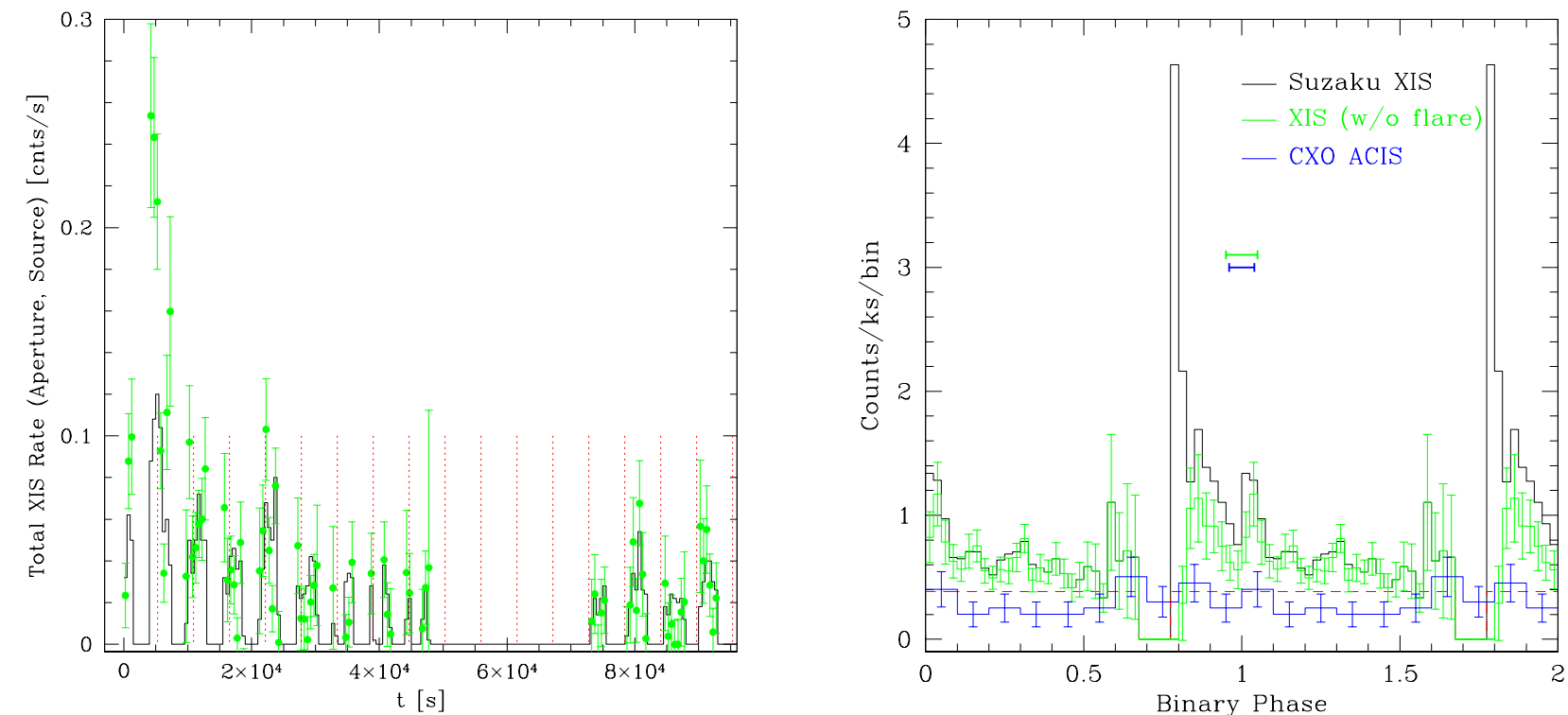

FIG. 2.- Left: Raw aperture count rate (histogram) and background- and aperture- corrected counts (points) during the Suzaku exposure. Times of optical flux maximum are marked. Right: Two periods of the X-ray orbital light curve of J1311.7-3429. Statistical errors are shown for the exposure-corrected bin count rate. For Suzaku, we show the folded data both with and without the bright flare. Horizontal error bars show the extrapolated phase uncertainty.

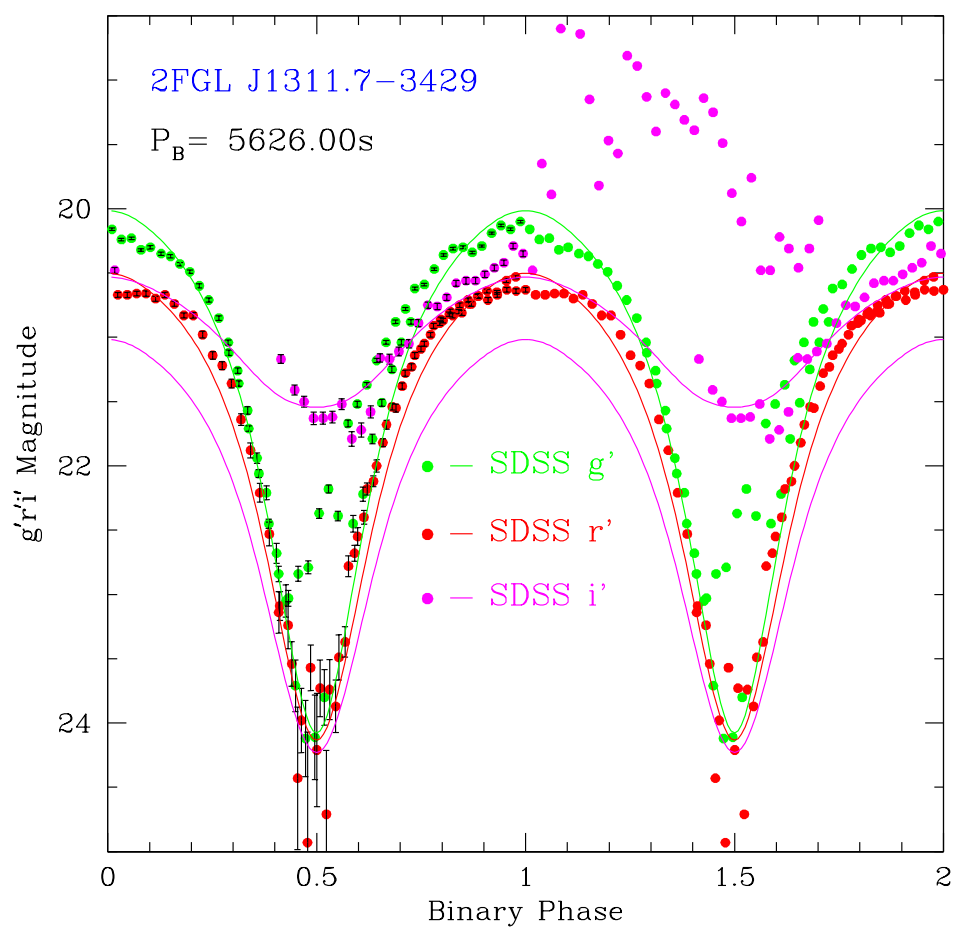

FIG. 3.- Folded SOAR $g^{\prime} r^{\prime} i^{\prime}$ light curves of J1311.7-3429 (two periods). Statistical errors are shown during the first period only and the large $i^{\prime}$ flare is plotted during the second period only. The curves represent a simple heated secondary model from ELC; a second copy of the $i^{\prime}$ curve with a constant flux offset is also shown for comparison.

(HEASARC NH tool), and fit for the power law index. Using CIAO the $C X O$ data fit to $\Gamma=1.3 \pm 0.5$. XSPEC fits to the Suzaku XIS0,3 give $\Gamma=1.6 \pm 0.8$. Fixing at $\Gamma=1.5$, we obtain a quiescent unabsorbed flux for the source of $f_{0.3-10 \mathrm{keV}}=2.4 \pm 0.6 \times 10^{-13} \mathrm{erg} \mathrm{cm}^{-2} \mathrm{~s}^{-1}$. A deeper exposure, allowing a better light curve and a orbit-dependent spectral study is clearly of interest.

\section{SYSTEM MODELING}

Figure 3 shows two periods of the SOI light curves folded on the ephemeris of $\S 2.1$. The large quasi-sinusoidal modulation shows that the light curve is dominated by heating of the secondary. The typical $g-r \approx-0.4$ near $\phi=0$ suggests $T_{\text {eff }} \approx 14,000 \mathrm{~K}$. By phase $\phi=0.35$ the color has reddened to $g-r>-0.1\left(T_{\text {eff }} \approx 8000 \mathrm{~K}\right)$. At flux minimum ( $\phi \sim 0.5$ ) large (up to $\Delta m=3$ ) fluctuations dominate. Since relatively small fluctuations can overwhelm the quiescent flux, these prevent any meaningful measurement of color at flux minimum.

We have attempted to model the 'quiescent' light curves with the ELC code (Orosz \& Hauschildt 2000). A formal fit is not indicated as there are many positive 'flare' excursions from any reasonable light curve. However comparison with a range of models shows that a few of the parameters are significantly constrained. For example, matching the characteristic $g^{\prime}-r^{\prime}$ at maximum requires heating by a primary luminosity (modeled here as an isotropic ' $\mathrm{X}$-ray' flux) of $\log \left[L_{x}(\mathrm{erg} / \mathrm{s})\right]=36.0 \pm 0.3$. Also, to obtain adequate modulation while avoiding an X-ray eclipse, requires $60^{\circ}<i<80^{\circ}$. At this point other parameters are poorly constrained, although large mass ratios and a near-Rochelobe filling secondary are preferred. However, the basic ELC model seems inadequate for a good fit; a particular challenge is to reproduce the relatively flat maxima and broad 'shoulders' in the $g^{\prime}$ and $r^{\prime}$ curves at $\phi \sim 0.25,0.75$. This may be due to limitations of the atmosphere tables. One can substantially improve the fit by modifying the limb-darkening law, but for blackbody surface spectra broad light curves (with adequate heating) require unphysical negative limb darkening coefficients. Another, more attractive, possibility is that the distribution of surface heating differs from the isotropic form assumed here; the pulsar may primarily heat a portion of the companion star. An illustrative set of curves is shown in Figure 3 , assuming a mass ratio $q=50$, orbital semi-major axis $a=0.9 R_{\odot}$, inclination $i=65^{\circ}$, heating flux $10^{36} \mathrm{erg} \mathrm{s}^{-1}$ and a $T_{\text {eff }}=4000 \mathrm{~K}$ companion reaching 0.99 of its Roche lobe radius. The observed $i^{\prime}$ flux evidently has a substantial added flux even before the bright flare, at the epoch observed.

\section{DISCUSSION}

At this short orbital period, the mean density in the secondary Roche lobe is $\left\langle\rho_{2}\right\rangle=46 \mathrm{~g} \mathrm{~cm}^{-3}$, so the secondary could be a main sequence star of $<0.15 M_{\odot}$. To reach 
this period, however, the system very likely passed through a common envelope phase, and would have a helium-rich core. The strong heating and near-Roche lobe filling suggest a substellar secondary, but additional observations are needed to determine the state of the secondary core and envelope.

A very rough estimate of the source distance can be made from the $g^{\prime}-r^{\prime}=-0.4$ color and $g^{\prime}=20.2$ flux at maximum, if we assume that the star nearly fills its Roche lobe at $\approx 0.46 a\left(M_{2} / M_{T o t}\right)^{1 / 3} \approx 0.1 R_{\odot}$. Such color indicates $T_{\text {eff }} \approx 14,000 \mathrm{~K}$ (type $\sim \mathrm{B} 6$ ), corresponding to main sequence $M_{g}=-0.43$ and $R \approx 3.0 R_{\odot}$. Correcting for the effective area and the small $\left(A_{g}=0.25\right)$ Galactic extinction in this direction, we get $d \approx 3.9(q / 50)^{-1 / 3} \mathrm{kpc}$. This is likely an overestimate as the secondary should be H-poor, and the temperature of the viewed hemisphere is far from uniform, even at maximum.

If we adopt the view that the $\mathrm{X}$ - and $\gamma$-ray emissions come from an energetic pulsar, we can make some additional estimates. Becker (2009) finds that pulsars have $L_{X} \approx 10^{-3} \dot{E}$, so from the observed X-ray flux we would infer a spindown luminosity $\dot{E} \approx\left(10^{3}\right) 4 \pi d^{2} 2.4 \times 10^{-13} \mathrm{erg} \mathrm{cm}^{-2} \mathrm{~s}^{-1} \approx$ $2.9 \times 10^{34} d_{k p c}^{2} \mathrm{erg} \mathrm{s}^{-1}$. Gamma-ray pulsars are also observed to follow a heuristic luminosity law $L_{\gamma, \text { heu }} \approx(\dot{E} \times$ $\left.10^{33} \mathrm{erg} / \mathrm{s}\right)^{1 / 2}$ (Abdo et al. 2010). This is related to the observed flux as $L_{\gamma}=4 \pi f_{\Omega} F_{\gamma} d^{2}$, where the beamingdependent correction factor is $f_{\Omega} \sim 0.7-1.3$ (Watters et al. 2009). Thus the observed $F_{\gamma}=6.2 \times 10^{-11} \mathrm{erg} \mathrm{cm}^{-1} \mathrm{~s}^{-1}$ gives a distance-dependent estimate for the spindown power of $\dot{E} \approx 5.5 \times 10^{34} f_{\Omega}^{2} d^{4} \mathrm{erg} \mathrm{s}^{-1}$. This is consistent with the $\mathrm{X}$-ray luminosity estimate when $d \approx 0.75 f_{\Omega}^{-1} \mathrm{kpc}$. However, if we match $\dot{E}$ to the ELC-estimated heating flux a distance of $\sim 2 \mathrm{kpc}$ is preferred.

The nature of the intermittent 'flaring' emission is at present unclear. Since the bands were observed sequentially, we do not even know the characteristic color. All we can infer are large increases above the quiescent flux (up to $\sim 5 \times$ in the optical; $\sim 6 \times$ in the X-ray, reaching a peak flux $\sim 6 \times 10^{32} d_{k p c}^{2} \operatorname{erg~s}^{-1}$ ). These are concentrated toward the heated side of the companion, but visible at all phases. Shocks in the wind stripping the companion could be the origin. However, since we do not know of such flaring activity on other well-studied black widow binaries, it is tempting to associate the activity with the unusually short $P_{b}$ of J1311. As has been noted (eg. Richards \& Albright 1993), low mass stars in close binaries often show enhanced coronospheric activity since tidal locking enforces rapid rotation, driving dynamogenerated magnetic fields. The other main ingredient for high magnetic activity is convection. This may suggest a low stellar-type mass for the companion. If sub-stellar, perhaps deep differential heating may drive the convection. A spectroscopic search for coronal line emission, or spectra during an outburst, could resolve the origin.

Additional data can greatly improve estimates of the system properties. Simultaneous multicolor photometry covering many periods and flaring events can help us understand the temperatures of the various emitting regions. A complete spectroscopic study of the secondary can place important constraints on the compact object mass and probe the state of the secondary photosphere. Spectra can also improve knowledge of the compact object's period, orbital epoch and semi-major axis, each of which are crucial for decreasing the parameter space to be searched for a $\gamma$-ray pulses. Of course, a $\gamma$-ray or radio pulsar ephemeris would produce a qualitative change in our knowledge of the system properties. We are pursuing all of these follow-up investigations.

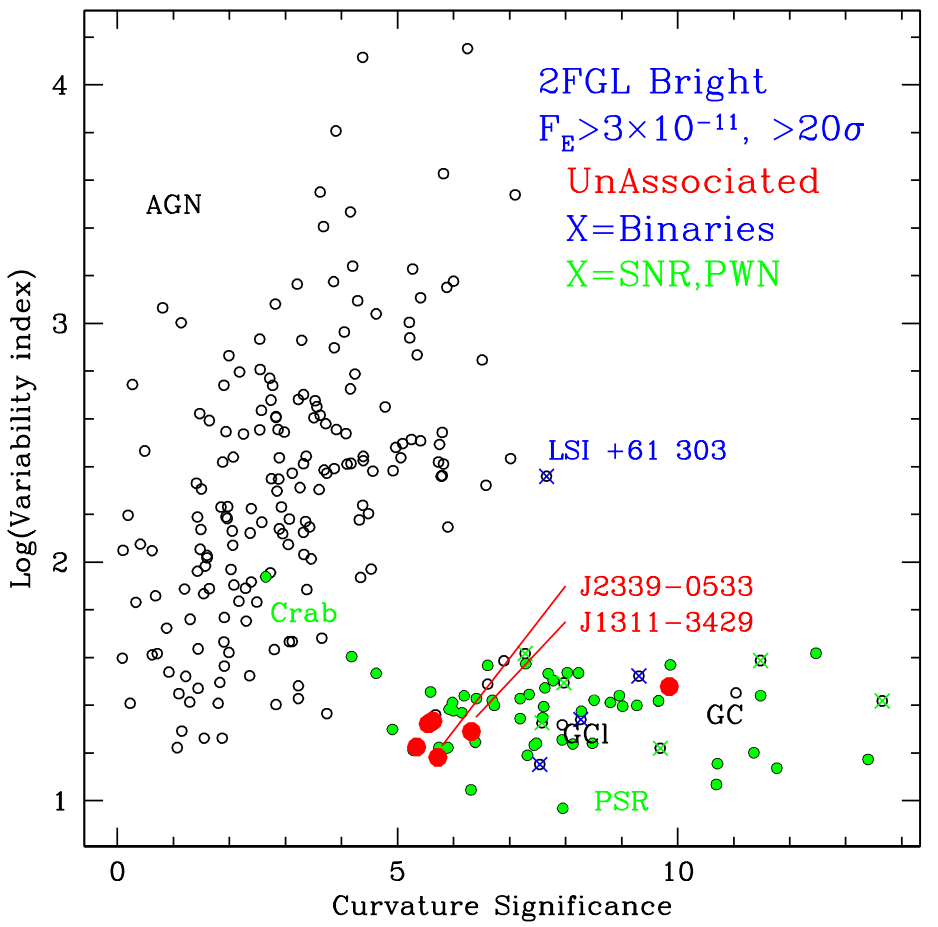

FIG. 4.-2FGL bright sources in the spectral curvature - variability plane. Note the excellent pulsar(PSR)/blazar(AGN) separation. Binaries, a globular cluster (GCl), the Galactic Center (GC) and the unidentified sources belong to the pulsar group.

We close with a comment on $\gamma$-ray pulsar population in the bright sample. Figure 4 shows these sources in the curvaturevariability plane. The pulsar-blazar separation is excellent; the only pulsar in the blazar region is the Crab, whose PWN contributes the variable power-law component. The pulsar region contains a few other source types: high mass binaries, a globular cluster, SNR, but each may harbor spin-powered pulsars. The unidentified sources, too, have significant curvature and low variability. Thus it is unsurprising that two of the six unidentified sources are good candidates for binary MSP, although they lack radio detection. The other unidentified sources may be similar or new source types. An interesting question is whether J2339-0533 and J1311-3429 represent the first of a new source class - radio quiet MSP. It is not yet clear that we need to appeal to such novelty: for both the optical gives evidence of a strong evaporative wind. It is entirely possible that radio emission is directed at Earth, but the pulse is scattered and dispersed to undetectability by this wind. Thus it remains possible that the MSP $\gamma$-ray beam lies within the radio emission zone - i.e. that all $\gamma$-ray MSP are radio detectable.

Even if these two sources represent a harbinger of a new source class we emphasize that this source class must be small, at least for the bright population, in contrast to some suggestions in the literature (Harding, Usov \& Muslimov 2005; Takata, Wang \& Cheng 2011). The statistics in our sample clearly make this case - of the 41 young pulsars in this set only $17(1 / 3)$ are radio selected. In contrast there are $12 \mathrm{MSP}$ in the bright sample, all radio pulsars. Thus even if all six unidentified sources prove to be radio-quiet MSP the radio loud subset will be at least $2 / 3$ of the $\gamma$-ray MSP, twice 
the fraction of the young pulsars. Thus, while J1311-3429 may have joined the 'black widow' club of pulsars evaporating their companions, it is not yet clear if there is a 'radio quiet MSP' club for it to join. And if such a society does exist, it is very select, indeed.
This work was supported in part by NASA grant NNX11AO44G.

The SOAR Telescope is a joint project of: Conselho Nacional de Pesquisas Cientificas e Tecnologicas CNPq-Brazil, The University of North Carolina at Chapel Hill, Michigan State University, and the National Optical Astronomy Observatory.

\section{REFERENCES}

Abdo, A.A. et al. 2009a, ApJS, 183, 46

Abdo, A.A. et al. 2009b, Science, 325, 840

Abdo, A.A. et al. 2010, ApJS, 187, 460.

Becker, W. 2009, ASSL, 357, 91

Harding, A.K., Usiv, V.V., \& Muslimov, A.G. 2005, ApJ, 622, 531

Kong, A. et al. 2012, ApJ, 747, L3.

Nolan, P.L. et al. 2012, ApJS, 199, 3

Orosz, J.A. \& Hauschildt, P.H. 2000, AA, 364, 265
Ray, P.S. et al. 2012, Proc. 2011 Fermi Symposium

Richards, M.T. \& Albright, G.E. 1993, ApJS, 88, 199

Romani, R.W. \& Shaw, M.S. 2011, ApJ, 743, L26

Takata, J, Wang, \& Y. Cheng, K.S. 2011, MNRAS, 415, 1827

Watters, K.P. et al. 2009, ApJ, 695, 1289 